\title{
Evaluación de la calidad edafológica y toxicológica de tres invernaderos con producción de hortalizas
}

Evaluation of edaphological and toxicological quality in three greenhouses with vegetable production

Dávila, L. M. del R ${ }^{1 \bowtie}$., Ramírez, H. T. ${ }^{1}$, Ortiz R. $\mathrm{H}^{1}$, Landero T. $\mathrm{I}^{1}$. y Enriquez R.V ${ }^{1}$

${ }^{1}$ Facultad de Ciencias Biológicas y Agropecuarias de la Universidad Veracruzana. Zona CórdobaOrizaba.

${ }^{\bowtie}$ Autor para correspondencia: charo davs@ hotmail.com

Recibido: 19/08/2016

Aceptado: 23/11/2016

\section{RESUMEN}

El objetivo de esta investigación fue determinar la calidad edafológica y toxicológica de tres de los invernaderos de la Facultad de Ciencias Biológicas Agropecuarias, los cuales son trabajados por los módulos de hortalizas y ornamentales. Las variables analizadas fueron: $\mathrm{pH}$, densidad, textura, materia orgánica (M.O), nitrógeno, potasio, fosforo, calcio y magnesio. La evaluación toxicológica se realizó cualitativamente evaluando la presencia y/o ausencia de: toxafeno, diclorodifeniltricloroetano (DDT), diclorodifenildicloroetano (DDD), endrin en presencia de dieldrin y/o aldrin, heptacloro y heptacloro en presencia de otros insecticidad organoclorados. Las variables se analizaron utilizando el programa estadístico SAS "Statistical Analysis System y la comparación de medias se realizó por la prueba de Tukey. Para las variables: pH, M.O, N, K, P, Ca y Mg en el análisis estadístico se reportaron diferencia significativa entre tratamientos, con respecto a la densidad no se encontró diferencia significativa en ningún tratamiento. $\mathrm{El}_{1} \mathrm{~T}_{1}$ y $\mathrm{T}_{2}$ (Invernadero de hortalizas) para las variables de $\mathrm{pH}$ y materia orgánica se obtuvieron resultados de 7.12-7.5 y $5.1 \%$ respectivamente los cuales se consideran ambientes propicios para uso agrícola, por otra parte se reportan en los tres invernaderos residuos tóxicos de DDT los cuales podrían deberse a la volatilidad y a su lenta degradación en el suelo.

Palabras clave: Suelos Análisis, Invernaderos, hortalizas. 


\begin{abstract}
The objective of this research was to determine the quality of soil science and toxicological three greenhouses Agricultural School of Biological Sciences, which are worked by modules vegetables and ornamentals. The variables analyzed were: $\mathrm{pH}$, density, texture, organic matter (M.O), nitrogen, potassium, phosphorus, calcium and magnesium. The toxicological evaluation was conducted qualitatively evaluating the presence and / or

absence of: toxaphene, dichlorodiphenyltrichloroethane (DDT), dichlorodiphenyldichloroethane (DDD), in the presence of dieldrin endrin and / or aldrin, heptachlor and heptachlor in the presence of other organochlorine insecticidal. The variables were analyzed using the SAS statistical program "Statistical Analysis System and comparison of means was performed by the Tukey test. For variables: pH, M.O, N, K, P, $\mathrm{Ca}$ and $\mathrm{Mg}$ in the statistical analysis significant difference between treatments were reported, with respect to density no significant difference was found in any treatment. The $\mathrm{T} 1$ and $\mathrm{T} 2$ (Greenhouse vegetables) for variables $\mathrm{pH}$ and organic matter results were obtained $7.12-7.5$ and $5.1 \%$ respectively which are considered enabling environments for agricultural use, on the other hand are reported in the three toxic waste greenhouses DDT which could be due to volatility and its slow degradation in soil.
\end{abstract}

Keywords: Soil Analysis, greenhouses, vegetables.

\section{INTRODUCCIÓN}

La calidad del suelo es producto de componentes químicos, físicos y biológicos y sus interacciones, puede ser definida como la capacidad del suelo para funcionar de una manera deseada tal como producir cultivos saludables. (Párr. et al, 1992, citado por López, 2002). La producción agrícola en cualquier sitio representa la interacción de suelo y factores meteorológicos; sistemas de cultivo, operaciones de siembra y labranza; uso de productos químicos, irrigación y, métodos de cosecha y disposición de residuos. Las actividades de producción agrícola pueden impactar los sistemas naturales alterando procesos de transporte: erosión hídrica y eólica, escurrimiento superficial, infiltración de agua en el suelo y transformación química, física y biológica. (López ,2002).

Considerando lo anterior, se plantea el objetivo siguiente:

Evaluar la calidad edafológica y toxicológica del suelo agrícola de los invernaderos de la Facultad de Ciencias Biológicas y Agropecuarias Campus Córdoba (FACBAC).

\section{MATERIALES Y MÉTODOS}

El trabajo se realizó en tres invernaderos de cultivos hortícolas en la Facultad de Ciencias biológicas y Agropecuarias de la Universidad Veracruzana ubicada en la localidad de

Revista Científica Biológico Agropecuaria Tuxpan 4 (2) 
peñuela, municipio de Amatlán de los Reyes Veracruz. México. Utilizando el Diseño Experimental Completamente al azar. La comparación de medias se realizó mediante la prueba de Tukey, utilizando el programa estadístico Statistical Analysis System (SAS). Por cada invernadero muestreado se tomaron 5 puntos, a los cuales se realizaron 3 repeticiones, los análisis y resultados fueron obtenidos en los laboratorios de suelos, agroindustrias y toxicología. Las variables de respuesta utilizadas fueron: $\mathrm{pH}$, Densidad, Materia orgánica, Nitrógeno, Potasio, Textura, Fósforo, Calcio, Magnesio y Análisis toxicológicos.

\section{RESULTADOS Y DISCUSIÓN}

Para la variable $\mathrm{pH}\left(\mathrm{H}^{+}\right)$analizado con $\mathrm{KCl}$, el análisis estadístico reporto diferencia significativa entre tratamientos, y se observó que el $\mathrm{T}_{2}$ el resultado fue 6.66 como valor menor y el mayor en el $\mathrm{T}_{1}$ con un $\mathrm{pH}\left(\mathrm{H}^{+}\right)$7.17. El cloruro de potasio $1 \mathrm{~N}$, tiene efecto en el $\mathrm{pH}$ del suelo, ya que las sustancias no tienen un $\mathrm{pH}$ neutro, sino, un poco acido, lo que hace que el resultado del $\mathrm{pH}$ de nuestro suelo se altere y se tome por unas unidades más acido. La densidad aparente es un dato muy valioso que se emplea para la detección en el suelo como son las capas endurecidas (Densidades mayores a $2 \mathrm{~g} / \mathrm{cm}^{3}$ ), las cuales provocan problemas de desarrollo radicular en los cultivos, la presencia de amorfos como el alofanó que comúnmente está relacionado con problemas de fertilización.

La materia orgánica es la principal variable que afecta las propiedades físicas y químicas del suelo. Los suelos con alto contenido de materia orgánica tienden a presentarmayorconductividad hidráulica, mayor porosidad, menor densidad aparente y menor compactación, lo que refleja en una medio ambiente propicio para la penetración radicular por ende en una alta fertilidad natural. En los suelos con altos nivel de materia orgánica (> 6.0) es donde se puede lograr los máximos rendimientos alcanzables para la variedad, clima y manejo del cultivo.

(J.Z. Castellanos. 2000). La concentración media de $\mathrm{N}$ inorgánico en el suelo se encuentra de $20-40 \mathrm{mg} \mathrm{Kg}^{-1}$. El potasio es un macro nutriente absorbido por las plantas en grandes cantidades, es el nutriente que menores problemas de disponibilidad presenta, a diferencia del nitrógeno y el fosforo, el

potasio no ocasiona problemas ambientales cuando sale del sistema

suelo, no es toxico y no causa eutrofización en los sistemas acuáticos. Los suelos orgánicos son deficientes en $\mathrm{K}$ pues contienen pocos minerales proveedores de este nutriente. El fosforo elemental $(\mathrm{P})$ no se encuentra en estado libre en la naturaleza porque se oxida muy fácilmente; el fosforo del suelo se clasifica en fosforo orgánico e inorgánico, dependiendo de la naturaleza de los compuestos. La forma orgánica se encuentra en el humus y la materia orgánica. La fracción inorgánica está constituida por compuestos de hierro, aluminio, calcio y flúor, entre otros. El $\mathrm{P}$ es un macro nutriente esencial para las plantas y el microorganismo, junto con el nitrógeno y el potasio. Puede ser un nutrimento limitante, ya que es un componente de los ácidos nucleídos y de los fosfolípidos. Los análisis de $\mathrm{P}$ sirven fundamentalmente para el control de la dosificación de productos químicos en tratamientos de agua o suelos, o como un medio para determinar que un sistema presenta contaminación por exceso de este compuesto. Las funciones del calcio 
contribuyen a la fertilidad del suelo, mejora la absorción de otros nutrientes por las raíces. Es esencial para los microorganismos que transforman los residuos de cultivo en materia orgánica, liberan nutriente $\mathrm{y}$ mejora tanto la estructura como la capacidad del suelo de almacenar agua. Este elemento fortalece la estructura de las plantas, coadyuva en varios sistemas enzimáticos. Ayuda a reducir la acidez del suelo beneficia a la planta al reducir el efecto negativo del aluminio y manganeso. (Resh, 1997) las concentraciones de $\mathrm{Ca}$ entre 5-10 Cmol

(+) $\mathrm{Kg}^{-1}$ representa una clasificación media de calcio. Uno de los papeles más importantes del magnesio es el que desarrolla en la formación de proteína. En caso de deficiencia de magnesio, la síntesis de proteína queda paralizada y la planta retrasa su crecimiento o desarrollo. El magnesio ( $\mathrm{Mg}$ ) ocupa la posición central de la molécula de la clorofila, la clorofila es un pigmento verde de la planta que interviene en la producción de materia orgánica utilizando la energía solar. Un adecuado suministro de $\mathrm{Mg}$ a las plantas intensifica claramente la actividad fotosintética de las hojas. Una concentración de 1.3-3.0 Cmol (+) $\mathrm{Kg}^{-1}$ de $\mathrm{Mg}$ representa una clase media.

En el tratamiento $\mathrm{T}_{1}$ El cultivo que se manejó en este invernadero fue el jitomate cid, empleando fertilización con Lombricomposta. Los químicos de control empleados fueron: Captan para la plaga tizón temprano (Alternaria solani) con dosis de $1.5-2.0 \mathrm{~kg} / \mathrm{ha}$. El captan es

un fungicida agrícola, por su composición porcentual presenta los siguientes ingredientes activos: $\mathrm{N}$ triclorometiltio-4-ciclohexeno-1,2-

dicarboximida, sus ingredientes inertes son: diluyente, humectante, dispersante y compuestos relacionados. En el tratamiento $\mathrm{T}_{2}$. los cultivos que se manejaron fueron el jitomate, variedad cid, zanahoria, apio, col, brócoli, para su fertilización se empleó abono orgánico lombricomposta, composta y fertilizante químico. Los químicos de control fueron el benlate para la plaga tiza, el cual es un fungicida sistémico con acción protectante y curativa, se absorbe a través de las hojas y raíces, con traslocación principalmente acropétala. Su ingrediente activo es benomyl en cual se descompone rápidamente vía hidrólisis, obteniéndose carbendazim (MBC) como principal producto de degradación y el 2aminobenzimidazole (2-AB) como producto de degradación menor. La vida media del benomyl bajo condiciones de suelo aeróbicas en laboratorio fue de aproximadamente DT50 $=19$ horas, mientras que la tasa de degradación del carbendazim es más lenta además de que su degradación es mediada por la acción microbiana del suelo. Por otro lado, el benomyl se puede considerar una molécula inmóvil, dado que tiene un coeficiente de adsorción de carbono orgánico promedio de $\mathrm{Koc}=1,857 \mathrm{ml} / \mathrm{g}$.

El tratamiento $\mathrm{T}_{3}$ localizado a la entrada de ésta institución, invernadero del módulo de ornamentales Los cultivos que se manejaron fueron el anturio, variedad fiesta, orquídeas, variedad shumburkia y chile jalapeño. Los químicos de control fueron el insecticida PLENUM, para la plaga pulgón (myzus persicae). Su ingrediente activo es el pymetrozine: 1 ,

2,3-triazin-3-(2H)-ona, 4,5-dihidro-6 metil-4(3-piridiilmetilen) amino. Es un insecticida selectivo del grupo químico triazinona asimétrica con un nuevo modo de acción. Tiene una acción sistémica y además es absorbido y traslocado en toda la planta, lo que permite controlar a los insectos cuando succiona la savia. Su acción es inmediata ya que los insectos permanecen vivos sin causar ningún 
daño, dejando de alimentarse y poco después mueren de inanición.

\section{CONCLUSIONES}

1. La densidad a pesar de obtener resultados dentro del rango en los tres invernaderos $\left(\mathrm{T}_{1}, \mathrm{~T}_{2}\right.$ y $\left.\mathrm{T}_{3}\right)$ en rangos de $1.24 \mathrm{~g} / \mathrm{cm}^{3}$ y $1.53 \mathrm{~g} / \mathrm{cm}^{3}$ se recomienda realizar periódicamente éste análisis, ya que al aumentar la densidad se puede presentar compactación impidiendo la adsorción y aireación del suelo.

2. En los otros nutrientes: nitrógeno (contenido $0.04 \mathrm{mg} \mathrm{Kg}^{-1}$ y 0.25 $\mathrm{mg} \mathrm{Kg}^{-1}$ ), fósforo (contenido 1.63 $\mathrm{mg} \mathrm{Kg}^{-1}$ y $10.67 \mathrm{mg} \mathrm{Kg}^{-1}$ ), calcio (contenido $1.18 \mathrm{Cmol}^{-1}\left(\mathrm{Kg}^{-1} \mathrm{y}\right.$ $4.34 \mathrm{Cmol}(+) \mathrm{Kg}^{-1}$ ) y magnesio (contenido $0.45 \mathrm{Cmol}^{(+)} \mathrm{Kg}^{-1} \mathrm{y}$ $1.50 \mathrm{Cmol}(+) \mathrm{Kg}^{-1}$ ), el contenido en los tres invernaderos se considera como clase baja, por lo que se recomienda considerar éstos resultados al inicio de producción de un cultivo.

3. Para otros trabajos similares realizar un análisis toxicológico cuantitativo, para la presencia de DDT, así como realizar una bitácora de control de los químicos empleados en cada uno de los invernaderos.

4. Los invernaderos 1 y $2\left(\mathrm{~T}_{1}\right.$ y $\left.\mathrm{T}_{2}\right)$ presentaron un $\mathrm{pH}$ de 7.35 considerados suelos con $\mathrm{pH}$ neutro y el invernadero $3\left(\mathrm{~T}_{3}\right)$ un $\mathrm{pH}$ de 7.50 suelo con $\mathrm{pH}$ moderadamente alcalino, concluyendo que son suelos en donde hay mayor disponibilidad de: potasio $(\mathrm{K})$, nitrógeno $(\mathrm{N})$, calcio $(\mathrm{Ca})$, magnesio $(\mathrm{Mg})$ y fósforo (P) ya que a esos $\mathrm{pH}$ presentan mayor solubilidad los nutrientes. $\mathrm{El} \mathrm{pH}$ con $\mathrm{KCl}$ los invernaderos 1,2 y $3\left(\mathrm{~T}_{1}, \mathrm{~T}_{2}\right.$ y $\left.\mathrm{T}_{3}\right)$ presentaron $\mathrm{pH}$ neutro, concluyendo que son suelos donde se puede encontrar una mayor disponibilidad de nutrientes antes mencionada.

5. La materia orgánica el invernadero $3\left(\mathrm{~T}_{3}\right)$ presentó 0.81 $\%$ considerado un suelo de clase bajo, el invernadero $1 \quad\left(\mathrm{~T}_{1}\right)$ presentó $1.98 \%$ clasificándose como suelo de clase media y el invernadero $2\left(\mathrm{~T}_{2}\right)$ presentó 5.12 $\%$ considerado un suelo de clase alto, concluyendo que el $\mathrm{T}_{2}$ presentó mayor contenido de materia orgánica lo cual presenta menor densidad aparente por ende mayor porosidad y menor contenido de arcilla.

6. Para el nitrógeno los invernaderos 1,2 y $3\left(\mathrm{~T}_{1}, \mathrm{~T}_{2}\right.$ y $\left.\mathrm{T}_{3}\right)$ presentaron contenidos muy bajos, por lo que se concluye que los tratamientos esta muy deficientes con respecto a este elemento vital para los cultivos.

7. El fosforo en los invernaderos $1 \mathrm{y}$ $3\left(\mathrm{~T}_{1} \mathrm{y} \mathrm{T}_{3}\right)$ presentaron $3.38 \mathrm{mg}$ ${ }^{-1} \mathrm{y} \quad 1.63 \quad \mathrm{mg} \mathrm{Kg}^{-1} \mathrm{Kg}$ respectivamente, por lo que se clasifica con un contenido deficiente, el invernadero 2 (T2) reporto $10.67 \mathrm{mg} \mathrm{Kg}^{-1}$, por lo que representa una clase baja. 


\section{LITERATURA CITADA}

Aislabie j, L. J.1995. A review of bacterial degradation of pesticides australian journal of soil research, 33: 925-942. https://doi.org/10.1071/SR9950925

Addiscott, T.1988.farmers, fertilizers, and the nitrate flood. New scienctist, 120:50-53.

Alarcón Castro A. A. 2012. Identificación de residuos tóxicos en hortalizas pepino

(Cucumis sativus), apio (Apium graveolens) y jitomate (Lycopersicum spp).tesis de licenciado en biología. Córdoba, ver. Facultad de ciencias biológicas y agropecuarias. $33 \mathrm{p}$.

Alexander, M.1997. Introduction to soil microbiology. 2nd.edition.john wiley and sons, New York, USA.

Arias J. A.C.2007. Suelos tropicales. universidad estatal a distancia, san José, costa rica, $188 \mathrm{p}$.

Barrow, C. J.1991. Land degradation, development and breakdown of terrestrial environments Cambridge University press. Cambrige, uk

Black A.L .1994.Residuos de plaguicidas en los alimentos, Organización de las naciones unidas para la agricultura y la alimentación. Roma. P. 28

Blum, W.H.1998. Basic concepts: degradation, resilience and rehabilitation. Pp. 1-16 en R. lal et al. (Eds) methods for assessment of soil degradation, advances in soil science.CRC press. Boca Raton, Florida, USA.

Cotí E, M. 2004. El potasio en los suelos y su rol en la producción agrícola, tesis, facultad de agronomía, universidad de buenos aires.

Cuesta Muños. P.A, Villaneda Vivas E. 2005. el análisis de suelo: toma de

muestra y recomendaciones de fertilización para la producción ganadera. Pp. 1-10.

Kookana R.S y aylmore L. A .G.1994. Estimating the pollution potential of pesticides to ground water. Australian journal of soil research,32: 1141-1155. https://doi.org/10.1071/SR9941141

López Falcón R.2002. Degradación del suelo: causas, procesos, evaluación e investigación. centro interamericano de desarrollo e investigación ambiental y territorial. Mérida, Venezuela.

Oldeman, L.R. 1988. Guidelines for general assessment of the status of

human-induced soil degradation. Working paper 88/4. International Soil Reference and Information Centre (ISRIC), Wageningen.

Ortiz, V.B., Ortiz, S. C. A. 1987. Edafología. Séptima edición. Editorial Departamento de suelos de la UACH, Chapingo, México, 128$129 \mathrm{pp}$.

Párr., J.F., R.I. Papendick, S.B. Hornik y R.E. Meyer.1992. Soil quality: attributes and relationship to alternative and sustainable agriculture. American journal of alternative agriculture, 7:2-3. https://doi.org/10.1017/S0889189300 https://doi.org/10.1017/S0889189300

Tisdale S.L, Nelson W.L .1985. Soil fertility and fertilizers. MC Milan publishing con., Inc, new York, USA. 


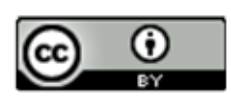

Este tex to está protegido por una licencia licencia Creative Commons 4.0.

Usted es libre para Compartir — copiar y redistribuir el material en cualquier medio o form ato-y Adaptar el documento —remezclar, transformar y crear a partir del material- para cualquier propósito,, incluso para fines comerciales, siempre que cumpla la condición de:

Atribución: Usted debe dar crédito a la obra original de manera adecuada, proporcionar un enlace a la licencia, e in dicar si se han realizado cambios. Puede hacerlo en cualquier form a razonable, pero no de form a tal que sugiera que tiene el apoyo del licenciante o 10 recibe por el uso que hace de la obra.

Resumencklicencia - Textocompletodelalicencia 